\section{Syndrome de Guillain-Barré et infection au SARS-CoV-2}

\section{Résumé}

Les auteurs rapportent l'observation de cinq patients ayant présenté un syndrome de Guillain-Barré (GBS) après une infection par le SARS-CoV-2. Ces cas ont été recensés entre le 28 février et le 21 mars 2020 dans trois hôpitaux du nord de l'Italie, parmi environ 1000 à 1200 cas admis pour une Covid-19 pendant la même période [1]. Les patients étaient âgés de 23 à 77 ans. À l'installation des troubles neurologiques, quatre patients avaient une PCR positive. Celui dont la PCR était négative a présenté des anticorps secondairement. Les premiers symptômes neurologiques étaient des paresthésies et un déficit des membres inférieurs avec aréflexie chez quatre patients et une diplégie faciale suivie d'ataxie sensitive et de paresthésies avec aréflexie chez le cinquième. L'EMG a montré une atteinte axonale chez trois patients et une atteinte démyélinisante chez deux. Une étude du liquide céphalo-rachidien n'a pas montré d'élément inflammatoire. L'imagerie par IRM a mis en évidence une prise de contraste des racines au niveau de la queue de cheval chez deux patients et des nerfs faciaux chez le patient présentant la diplégie faciale. Tous les patients ont présenté des symptômes extra neurologiques dans les cinq à dix jours précédents : fièvre pour $3 / 5$, autres symptômes pour tous les patients (toux: 4/5, mal de gorge: $1 / 5$, hypo ou anosmie: $3 / 5$ ), avec dans tous les cas une lymphopénie et une CRP élevée. L'imagerie pulmonaire a montré une pneumopathie interstitielle dans quatre cas sur cinq, et trois patients ont nécessité une ventilation assistée. Les cinq patients ont reçu des immunoglobulines intraveineuses et un a été traité secondairement par échanges plasmatiques. L'évolution après 4 semaines après le traitement a été favorable pour le patient présentant la diplégie faciale. Deux autres patients continuent de bénéficier d'une rééducation pour déficit moteur persistant, et deux sont toujours sous assistance ventilatoire.

\section{Lu pour Vous}

\section{Clinique}

Françoise Chapon

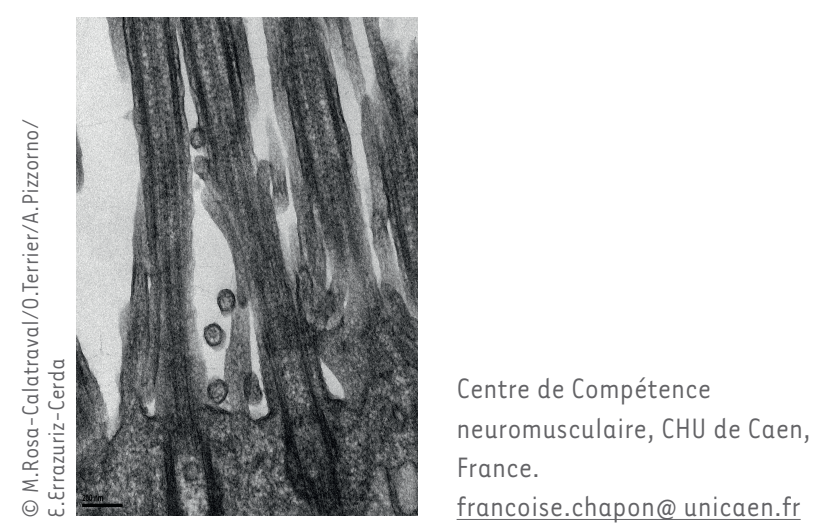

\section{Commentaires}

Cet article est le premier rapportant plusieurs cas de GBS associés à la Covid-19. La relation entre les deux affections apparaît nette étant donné le délai bref d'installation des troubles neurologiques après les premiers signes d'infection. Une neuromyopathie de réanimation a été évoquée mais peut être raisonnablement éliminée, celle-ci survenant habituellement plus tardivement. II faut souligner la fréquence, même sur ce petit effectif, des signes d'atteinte axonale. L'évolution apparaît, sur cette série, peu favorable même si le suivi est effectué sur une période trop courte pour en tirer des conclusions. D'autres publications sont parues antérieurement ou concomitamment à celleci : la première en date: Zhao et al. [2], puis plus de 50 publications sont parues sur cette association GBS-Covid-19 dont la plupart rapporte un seul nouveau cas avec parfois une présentation clinique particulière (syndrome de Miller Fisher, diplégie faciale, dysautonomie, forme pédiatrique...) sur un total de 102 publications si l'on retient les publications générales traitant des «complications /associations neurologiques et Covid-19». Des revues regroupent les données : celle de Guijarro-Castro et al. [3] reprend les caractéristiques de 16 cas de GBS/Covid19, celle de De Sanctis et al. [4] porte sur 18 cas recensés de novembre 2019 à mi-mai 2020, avec ici une prédominance d'atteintes démyélinisantes. Les revues les plus importantes sont celles d'Uncini et al. [5] (42 cas de janvier à juin) et d'Abu-Rumeileh et al. [6] (73 cas recensés de janvier à mi-juillet), et incluant 52 références. Ces deux dernières synthèses s'accordent pour une description le plus souvent de formes classiques de GBS avec une prévalence élevée de la forme classique sensitivomotrice de polyradiculoneuropathie inflammatoire aiguë, démyélinisante ( $80,5 \%$ des cas pour [5]) -, avec une dissociation albumino-cytologique dans plus de $70 \%$ des cas et un pronostic favorable après traitement par immunoglobulines intraveineuses. Le 
grand nombre de cas rapportés et la prévalence élevée de cas de la Covid-19 asymptomatiques rend nécessaire le dépistage systématique de ce virus afin de pouvoir évaluer plus précisément l'incidence de cette association et les particularités de ces cas de GBS par rapport aux cas GBS non associés à la Covid-19.

Guillain-Barré syndrome associated with SARS-CoV-2

\section{LIENS D'INTÉRÊT}

L'auteure déclare n'avoir aucun lien d'intérêt concernant les données publiées dans cet article.

\section{RéFÉRENCES}

2. Zhao H, Shen D, Zhou H, et al. Guillain-Barré syndrome associated with SARS-CoV-2 infection: causality or coincidence? Lancet Neurol $2020 ; 19$ : $383-4$

3. Guijarro-Castro C, Roson-Gonzales M, Abreu A, et al. Guillain-Barré syndrome associated with SARS-CoV-2 infection. Comments after 16 published cases. Neurologia $2020 ; 35: 412-5$.

4. De Sanctis $P$, Doneddu PE, Vigano L, et al. Guillain-Barré syndrome associated with SARS-CoV-2 infection. A systematic review. Eur J Neurol 2020, Aug 5;10.1111/ene.14462. doi: 10.1111/ene.14462.

5. Uncini A, Vallat JM, Jacobs BC. Guillain-Barré syndrome in SARS-CoV-2 infection: an instant systematic review of the first six months of pandemic. J Neurol Neurosurg Psychiatry $2020 ; 91$ : 278-84.

6. Abu-Rumeileh S, Abdelhak A, Foschi M, et al. Guillain-Barré syndrome spectrum associated with COVID-19: an up-to-date systematic review of 73 cases. J Neurol 2020 Aug 25: 1-38.

1. Toscano G, Palmerini F, Ravaglia S, et al. Guillain-Barré syndrome associated with SARS-CoV-2.

N Engl J Med 2020 ; 382 : 2574-6.

\section{www.myobase.org}

Catalogue en ligne disponible gratuitement sur Internet publié par l'AFM-Téléthon.

Retrouvez facilement toutes les références bibliographiques sur les maladies neuromusculaires, les situations de handicap qu'elles génèrent et leurs aspects psychologiques.

\section{Myobase donne un} accès libre à $75 \%$ du fonds documentaire collecté depuis 1990 représentant plus de 40000 références spécifiques du domaine des maladies neuromusculaires.

$>$ articles de la littérature biomédicale et psycho-sociale

\section{$>$ livres, thèses}

$>$ guides d'associations et rapports institutionnels d'agences internationales

\section{$>$ brèves en français,} synthèses des articles médico-scientifiques internationaux les plus pertinents

\section{$>$ publications}

AFM-Téléthon destinées aux professionnels de santé ou aux personnes atteintes de maladie neuromusculaire et à leur entourage

\section{UN OUTIL ERGONOMIQUE, UNE INTERFACE BILINGUE}

\section{- Laissez-vous guider par les tutoriels}

- Lancez une recherche et affinez votre sélection grâce aux filtres

\section{TOUT MYOBASE \\ Rechercher... Ok \\ Recherche avancée \\ Histo FILTRES \\ Type de document \\ Article [3443] \\ Publication AFM [176] \\ Thèse/Mémoire [107] Brève [102]}

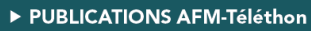

\section{$\checkmark$ BRÈVES}

๑ DOCUMENTS DE SYNTHÈSE

INSTITUT DES BIOTHÉRAPIES PUBLICATIONS

- Partagez les résultats de votre recherche

\section{UN ACCÈS facile et simple}

Rechercher avec des opérateurs :

- guillemets pour une expression

"maladie de pompe"

- + pour signifier ET, et retrouver tous les documents contenant les deux mots "fauteuil +électrique"

- - pour signifier NON et enlever le mot de la recherche:

"autonomie -établissement"
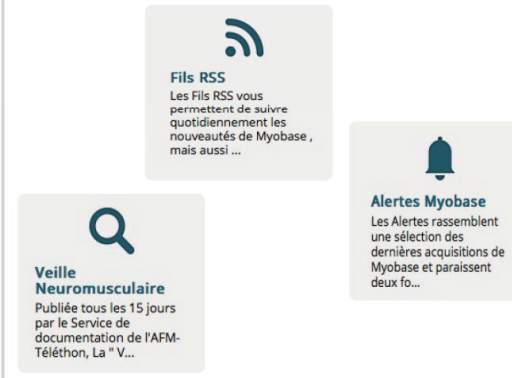

- Cliquez sur l'onglet

thématique qui vous convient (haut de la page d'accueil)

- Créez vos alertes personnalisées en ouvrant un compte personnel

- Téléchargez la Veille Neuromusculaire

- Abonnez-vous aux flux RSS 